\title{
IMPACT OF TRANSVERSE IRREGULARITIES AT THE PHOTOCATHODE ON THE PRODUCTION OF HIGH-CHARGE ELECTRON BUNCHES*
}

\author{
M. Rihaoui, C.L. Bohn ${ }^{\dagger}$, Northern Illinois University, DeKalb, IL,60115, USA \\ P. Piot, Northern Illinois University DeKalb, IL 60115, \& Fermilab, Batavia, IL 60510, USA \\ J.G. Power, ANL, Argonne, IL 60439, USA
}

\begin{abstract}
The properties of electron beams produced in a photoinjector are strongly dependent on the initial conditions, i.e. the photocathode drive laser shape and its uniformity. We explore the impact of well-defined transverse laser perturbations on the evolution of the electron beam both in configuration and velocity spaces and especially investigate how certain types of perturbations evolve as the beam propagates in the Argonne Wakefield Accelerator facility. Numerical simulations performed with IMPACT-T are presented. Finally preliminary experimental results aimed at validating our simulations are discussed.
\end{abstract}

\section{INTRODUCTION}

Phototoinjectors are designed to produce electron beams with high phase space density. The intricate beam dynamics in a photoinjector has led to the development of elaborated simulation tools capable of simulating the evolution of the particle distributions. Most of these programs are, to some extent, benchmarked in a root-mean-square sense. In this paper we attempt to perform more refined validation of beam dynamics programs. We perturb the initial transverse distribution of the photoemitted electron bunch in a welldefined manner and study the evolution of this density perturbation as the beam is transported along the accelerator. The perturbation is controlled with an appropriate control of the photocathode drive laser transverse profile.

\section{AWA FACILITY}

\section{Overview}

The Argonne Wakefield Accelerator (AWA) [1] is designed to produce high charge and low emittance bunches for advanced accelerator R\&D. The backbone of the AWA accelerator is a high-charge photoemission source based on a $1+1 / 2$ cell rf-gun operating on the $\mathrm{TM}_{010, \pi}$ at $f=$ $1.3 \mathrm{GHz}$. A $3.5 \mathrm{ps}(\mathrm{rms})$ ultraviolet $(\lambda=248 \mathrm{~nm})$ laser pulse impinges a Magnesium photocathode and electron bunches with maximum charge of $100 \mathrm{nC}$ are photoemitted. The nominal electric field amplitude on the photocathode is approximately $80 \mathrm{MV} / \mathrm{m}$ resulting in a beam energy, at the exit of the gun, of $8 \mathrm{MeV}$. The gun is surrounded

\footnotetext{
* Work supported by U.S. Department of Energy under Contract No. DE-FG02-04ER41323 with Northern Illinois University.

${ }^{\dagger}$ deceased
}

by three solenoidal magnetic lenses termed as bucking, primary (or focusing), and secondary (or matching) solenoids. After the rf-gun the beam is further accelerated to $15 \mathrm{MeV}$ with a $1.3 \mathrm{GHz}$ standing-wave booster cavity. Downstream of the booster cavity, the beamline consists of quadrupoles, dipole correctors, a spectrometer and an extensive suite of beam diagnostics.

\section{Optimum Settings}

We carried a series of numerical optimization with the goal of minimizing the transverse emittance at $z=7 \mathrm{~m}$ from the photocathode. The optimizations were performed using ASTRA [6] and the optimized values found were used as an input parameters for more detailed (and CPU-intensive) simulations in IMPACT-T [7]. We used the SDDSOPTIMIZE [3] optimizer and wrote python-based wrapper [5] to utilize SDDSOPTIMIZE in conjunction with ASTRA. In Table 1 we summarize the operating parameters resulting in a minimum transverse emittance for two cases of bunch charges.

Table 1: Optimized values for the AWA facility at $z=7 \mathrm{~m}$ (end of photoinjector).

\begin{tabular}{lcc}
\hline \hline$Q(\mathrm{nC})$ & 0.1 & 1 \\
\hline$\sigma_{x}(\mathrm{~mm})$ & 0.5 & 0.504 \\
$\phi(\mathrm{rf}$ degrees $)$ & 55.2 & 55.3 \\
$B_{\text {bucking }}(\mathrm{T})$ & 0.1171 & 0.1318 \\
$B_{\text {primary }}(\mathrm{T})$ & -0.1182 & -0.1390 \\
$B_{\text {secondary }}(\mathrm{T})$ & -0.4585 & -0.4983 \\
$\varepsilon_{x}(\mu \mathrm{m})$ & 0.704 & 1.944 \\
\hline \hline
\end{tabular}

The optimized values obtained for $Q=1 \mathrm{nC}$ were used in IMPACT-T to study the difference in beam parameter for a transversely uniform and for a measured photocathode drive laser spot; see Fig. 1. The initial non-uniformities lead to emittance growth.

\section{ASTRA $v s$ IMPACT-T}

IMPACT-T is a 3D tracking algorithm while ASTRA incorporate a cylindrical symmetric mesh for the space charge calculation. A comparison between the two codes is possible if we consider a uniform cylindrical symmetric initial distribution. Such a comparison for $Q=1 \mathrm{nC}$ is shown in Fig. 2. The agreement is decent and the observed 

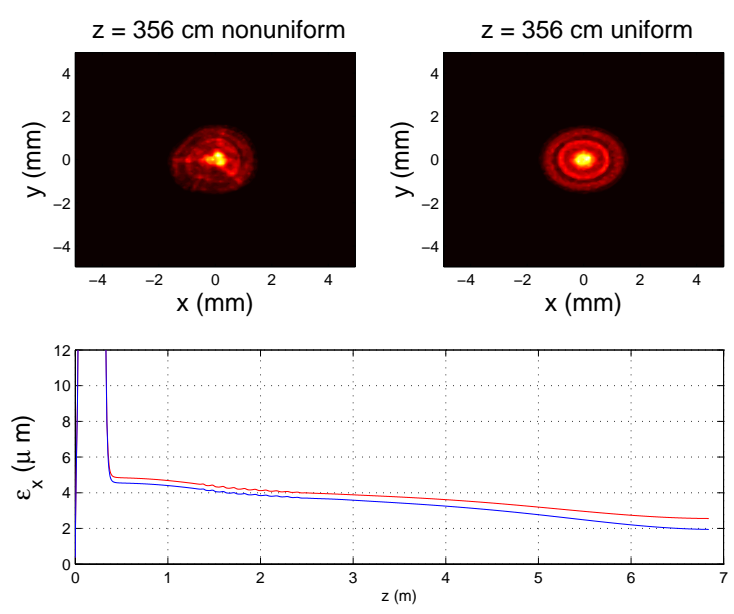

Figure 1: Comparison of beam transverse density at $z=$ $356 \mathrm{~cm}$ for a uniform (top left) and realistic (top right) initial laser beam distribution. Associated transverse emittance evolution along the accelerator (bottom) [realistic laser (red line) uniform laser (blue line)].

small differences might be attributed to the difference in the space charge algorithm and emission algorithm. When the space charge routine is turned off the agreement between the two codes is perfect.
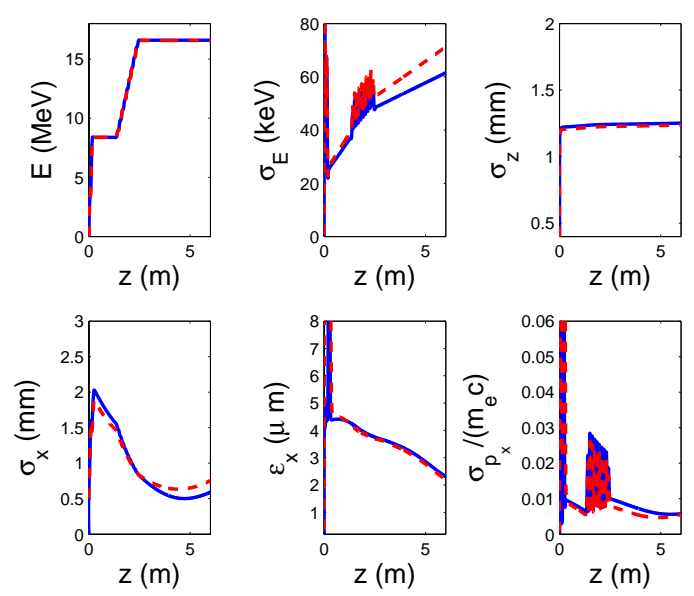

Figure 2: Comparison of beam parameters obtained with ASTRA (blue solid line) and IMPACT-T (red dashed line) when space charge effects are included. The parameters are: $E$ is the energy of the beam, $\sigma_{E}$ is the rms energy spread, $\sigma_{z}$ is the bunch length, $\sigma_{x}$ is the beam spot size on the cathode, $\epsilon_{x}$ and $\sigma_{p_{x}}$ are respectively the rms transverse emittance and momentum spread.

\section{SIMULATION OF PERTURBED BEAMS}

The photocathode drive laser sets the initial conditions for the electron beam parameters and tailoring the laser distribution in a predefined way to linearize the space charge force will improve the beam brightness. The transverse density of the photocathode drive laser can, in principle, be easily shaped using a properly designed optical transport line. However non-uniformities in the laser spot are unavoidable. Typically, non-uniformities result from the frequency conversion process in nonlinear crystal. The initial macroparticle distribution used in the simulations was generated by a MATLAB [9]-based program that gives a six-dimensional phase space distribution with position coordinates generated from the measured laser beam distribution on the photocathode [8]. All the simulations presented in this Section uses the AWA optimized settings reported in the previous Section.

We first consider a quincunx distribution as used in previous work on space-charge-dominated beam physics [10]. Such a pattern can easily be generated by intercepting the photocathode drive laser with a mask. According to Reference [11], this distribution is motivated by practical applications like heavy ion fusion where a large beam with low emittance is required and it is difficult to accelerate the beam in one focusing channel. The diameter of each beamlet is $1 \mathrm{~mm}$ and the total charge is $1 \mathrm{nC}$. We also considered dipole-type $(\zeta=1)$ and the quadrupole $(\zeta=2)$ perturbation on the initial density perturbation:

$$
\rho(r, \theta)=\rho_{0}(r, \theta)(1+m r \cos (\zeta \theta))
$$

where $m$ is the strength of the initial perturbation,, $\rho_{0}$ is the unperturbed distribution (i.e. considered to be radial uniform here). This type of perturbation can be produced by intercepting the laser beam with custom-made neutral density ultraviolet filter.

Figure 3 presents the transverse density evolution along the accelerator associated to the three aforementioned distribution. The evolution of the quincunx distribution show intricate series of complex that depends on when and where the beamlet crossed over. The dipole perturbation eventually smears out and the distribution "forgets" about the initial perturbation. The quadrupole perturbation is also washed out but later in the beamline higher harmonic perturbations seems to appears (see $z=470 \mathrm{~cm}$ ). A fourier analysis of the pattern for the quadrupole and dipole perturbation is presented in Fig. 4

\section{PRELIMINARY EXPERIMENT}

We conducted a preliminary set of experiment aimed at verifying the possible usefulness of the method described in the thesis and getting familiar with the operation of the AWA accelerator. Our experiment should be considered more as a proof-of-principle experiment rather than a detailed study. For this first experiment we used a mask consisting of an aluminum plate with six holes bored in it. The mask was located in the accelerator enclosure and intercepted the photocathode drive laser $50 \mathrm{~cm}$ before the photocathode. A uv-sensitive CCD camera was used to record generated pattern at the virtual cathode; see Fig. 5 top row, 


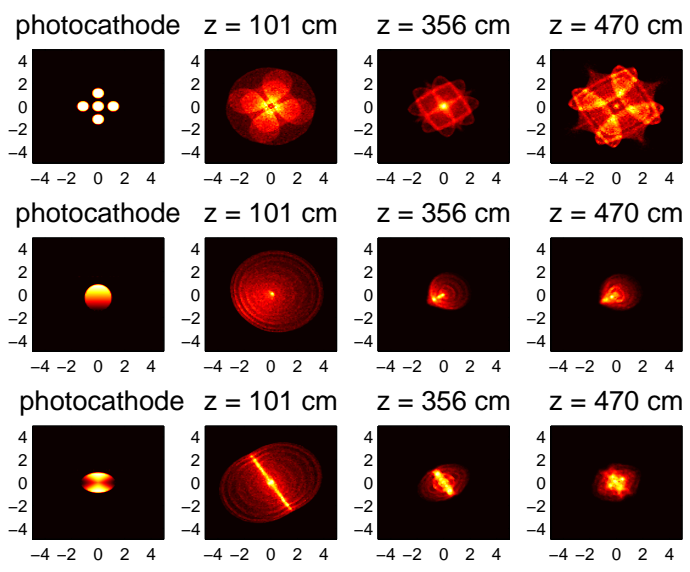

Figure 3: Transverse beam distributions at different locations along the AWA obtained by tracking an initial quincunx distribution (top row), dipole (middle row) and quadrupole (bottom row) perturbations.
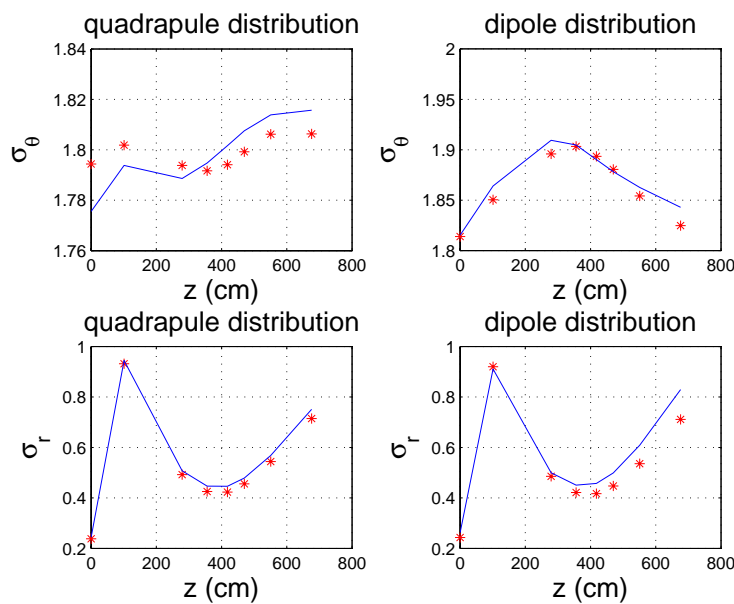

Figure 4: Top two plots for the quadrupole and the dipole for $\sigma_{\theta}$. Bottom two plots for $\sigma_{r}$, red stars for $\mathrm{m}=0.01$ and blue line for $\mathrm{m}=0.02$.

Some crude alignment was performed in order to align the mask such that the laser beam hits the center of the photocathode. The solenoids were varied and picture were taken for different excitation currents. The E-field amplitude in the rf-gun needed to be decreased: for the high precision measurement needed in this experiment we could not tolerate the dark current emitted at $80 \mathrm{MV} / \mathrm{m}$; see Tab.2.

Figure 5 compares simulations done for six beamlets with IMPACT-T along with the experiment. Overall the agreement is not perfect and this might be attributed to the not yet well defined experimental conditions or an imperfect in the space-charge routine. Qualitatively some features predicted by the simulation are reproduced in the experiment, for instance the beamlets shapes are no more circular and eventually acquire a triangular character; YAG2 and YAG3 in Figure 5.
Table 2: Experimental settings used during the preliminary experiment done at the AWA facility.

\begin{tabular}{ccc}
\hline \hline Input Parameter & Value & Unit \\
\hline Electric field on cathode & 54 & $\mathrm{MV} / \mathrm{m}$ \\
Launching Phase & 50 & $\mathrm{rf} \mathrm{degrees}$ \\
Electric field in the linac & 0 & $\mathrm{MV} / \mathrm{m}$ \\
Bucking solenoid field & 0.1294 & $\mathrm{~T}$ \\
Focusing solenoid field & -0.1362 & $\mathrm{~T}$ \\
Matching solenoid field & -0.3639 & $\mathrm{~T}$ \\
Laser pulse duration (rms) & 3.5 & $\mathrm{ps}$ \\
Laser spot size on cathode (rms) & 2 & $\mathrm{~mm}$ \\
Charge & 1 & $\mathrm{nC}$ \\
\hline \hline
\end{tabular}
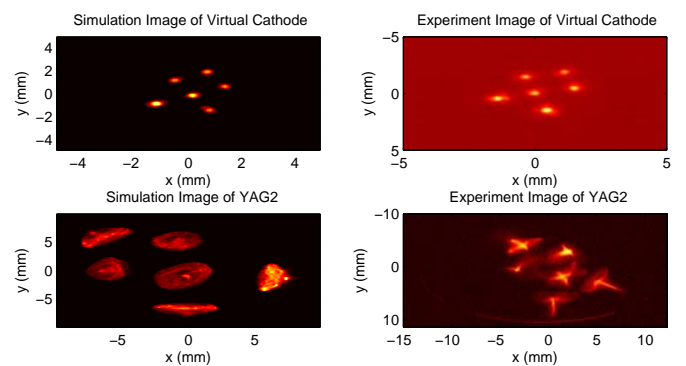

$\begin{array}{ccc}-5 & 0 & 5 \\ \text { Simulation Image of } Y A G 3\end{array}$
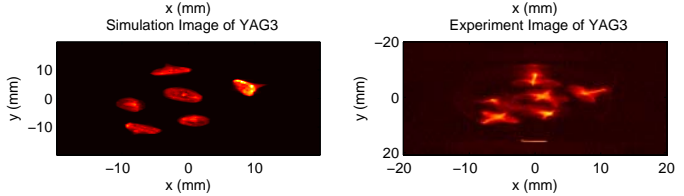

Figure 5: Simulated transverse beam density associated to a six-beamlet configuration of the photocathode drive laser (left) and corresponding measured distribution (right).

\section{REFERENCES}

[1] http://www.hep.anl.gov/pmalhotr/AWA/index.html.

[2] J. Power, Proceedings of the 2003 Particle Accelerator Conference, Portland, OR, 2432 (2003).

[3] H. Shang et al., Proceedings of the 2003 Particle Accelerator Conference, Portland, OR (2003), 3470 (2003).

[4] M. Borland, Proceedings of the 1995 Particle Accelerator Conference, Dallas, TX (1995), paper WAE11 (1995)

[5] http://www.python.org/

[6] K. Flottmann,AstRA: A Space Charge Tracking Algorithm, available at http://desy.de/ mpyflo.

[7] J. Qiang, et.al., Phys. Rev. ST A\&B 9, 044204 (2006).

[8] M. Rihaoui, MS thesis, Northern Illinois University (2007).

[9] http://www mathworks.com

[10] M. Reiser et.al., Phys. Rev. Lett. 61, p. 2933 (1988).

[11] I. Haber et.al., Phys. Rev. A, 44 (1991). 